\title{
On the Impact of Alterations on Face Photo Recognition Accuracy
}

\author{
Matteo Ferrara, Annalisa Franco, Davide Maltoni, and Yunlian Sun \\ Department of Computer Science and Engineering, University of Bologna, Cesena, Italy \\ \{matteo.ferrara, annalisa.franco, davide.maltoni, \\ yunlian.sun2\}@unibo.it
}

\begin{abstract}
This work is framed into the context of automatic face recognition in electronic identity documents. In particular we study the impact of digital alteration of the face images used for enrollment on the recognition accuracy. Alterations can be produced both unintentionally (e.g., by the acquisition or printing device) or intentionally (e.g., people modify images to appear more attractive). Our results show that state-of-the-art algorithms are sufficiently robust to deal with some alterations whereas other kinds of degradation can significantly affect the accuracy, thus requiring the adoption of proper detection mechanisms.
\end{abstract}

Keywords: ICAO, eMRTD, face recognition, image alteration, digital beautification.

\section{Introduction}

In recent years traditional identity documents have been replaced by electronic documents able to store biometric features to be used for machine-assisted identity verification [1] [2]. With the Berlin resolution (2002), the International Civil Aviation Organization (ICAO) selected the face as the primary globally interoperable biometric characteristic for machine assisted identity confirmation in electronic Machine Readable Travel Documents (eMRTD) [3].

In order to facilitate the automatic identity verification process, the images stored in an ICAO compliant electronic document have to fulfill very restrictive quality standards, i.e. no elements that could compromise the recognition accuracy should be present. A number of indications about the geometric and photometric properties of the face images to be used in e-documents are given in the ISO/IEC 19794-5 standard following the guidelines initially proposed by ICAO. For instance the subject should have a well-controlled pose, a proper lighting, a natural expression, no accessories that could partially occlude some important facial characteristics, etc...

Some of the countries issuing e-documents, acquire the face image of the subject at the enrollment station with a digital camera. Other countries (e.g., Italy) require the user to provide a printed face photo (ID format) to the issuing authority. While in the first case it is enough to verify (manually or in machine-assisted way) ISO/IEC 
19794-5 compliance [4], in the second case a further validation should be done to ensure that the face in the printed photo has not (intentionally or unintentionally) been altered.

In fact, several problems may raise in different scenarios if an altered face image were included in the document:

- $\quad$ in a verification scenario, for instance in an automatic gate in an airport, the alterations may determine a high rate of false rejections, i.e. the system would not recognize the person thus making necessary the human intervention;

- $\quad$ in a watch-list scenario, where a list of subjects wanted by the police has to be checked in order to raise proper alarms, the presence of altered face images in the documents could imply missing the suspect that, in this case, could intentionally alter the face image to reduce the probability of being identified.

More in detail, possible alterations includes: i) intentional digital alteration of the face image, for instance to obtain a beautification; ii) geometric alterations introduced by the acquisition device or a bad printing process.

This paper presents a study of the effects of digital image alteration on face recognition performance. For the experimentations we used three reference recognition approaches: two commercial systems and one algorithm at the state-of-the-art are used.

The paper is organized as follows: in section 2 the digital alterations considered in the experiments are presented, section 3 details the experiments carried out and finally section 4 draws some conclusions.

\section{Digital Face Image Alteration}

Digital alterations of face images can be broadly organized in two categories: geometric and appearance-based. The first category includes transformations that are typically introduced by either the acquisition or the printing device (e.g. barrel distortion or change in the image aspect ratio); this kind of alterations are usually unintentional. The second kind of transformation includes all the alterations that can usually be performed by some image processing software. Such alterations are usually intentionally introduced to make the image more attractive; moreover several software applications that allow to simulate plastic surgery interventions are now available. In this section, we will investigate different types of alterations which are likely to be found in practical cases.

\subsection{Alterations}

For each kind of alteration, we modify the original image at different levels (i.e., with different strength). We use a parameter $p$ to describe the strength. A large value of $p$ denotes a more significant alteration. 


\section{Barrel distortion}

Barrel distortion is one of the most common types of lens distortions and represents the typical defect that could be introduced by a low quality acquisition device. In this transformation, a barrel distortion with a strength of $p$ is imposed on the original image while preserving the image size. The approach described in [5] has been adopted to implement this transformation. The value of $p$ is increased in steps of $2 \%$ from $10 \%$ to $20 \%$, i.e. $p \in\{0.10,0.12,0.14,0.16,0.18,0.20\}$. An altered image obtained applying the barrel distortion with $p=0.20$ is shown in Fig. 1 (b).

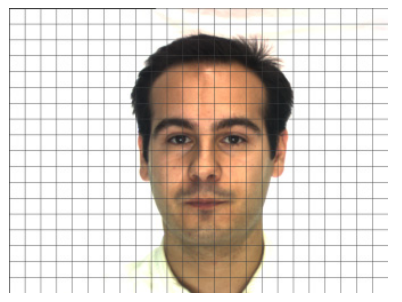

(a)

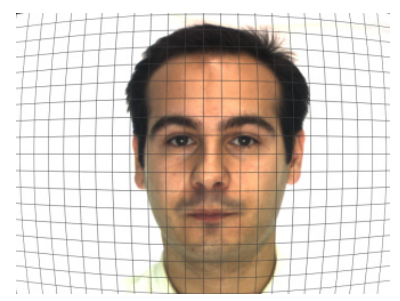

(b)

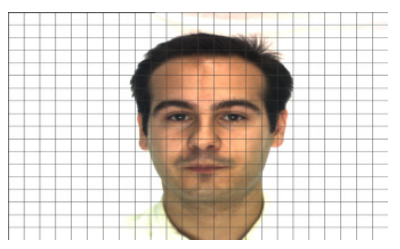

(c)

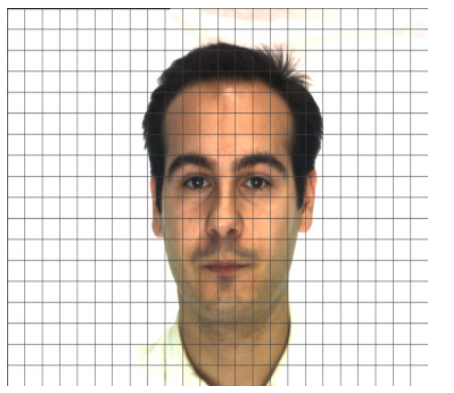

(d)

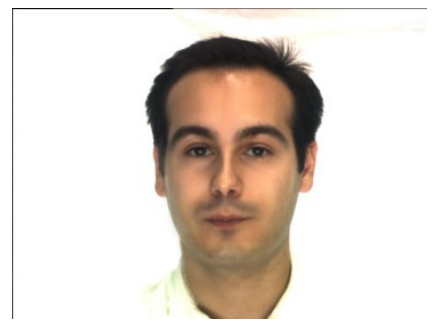

(e)

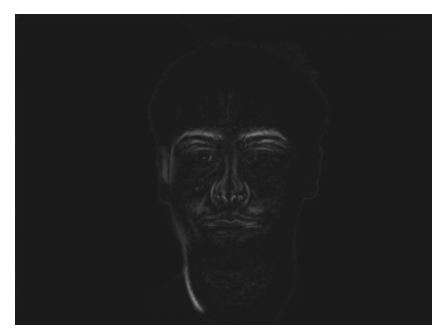

(f)

Fig. 1. Example of each alteration. (a) Original image. (b) Altered image with barrel distortion. (c) Altered image with vertical contraction. (d) Altered image with vertical extension. In a), b), c) and d) a squared grid is superimposed to the image to better highlight the effects of geometric alterations. (e) Altered image with digital beatification. (f) Pixel difference between original a) and digitally beautified image e). 


\section{Vertical Contraction}

In this alteration, we vertically compress the image while keeping the width fixed. In particular we reduce the original height by a multiplying factor of $(1-p)$. The values of $p$ remain the same to those in the barrel distortion. Fig. 1 (c) shows an altered image after vertical contraction with $p=0.20$.

\section{Vertical Extension}

On the contrary, in vertical extension, the height is increased by a multiplying factor of $(1+p)$ while keeping the width invariable. Here too we increase the strength of extension from $10 \%$ to $20 \%$ in a step of $2 \%$. An altered image after vertical extension with $p=0.20$ is shown in Fig. 1 (d). This alteration (and the previous one) which are essentially a modification of the face aspect ratio, could be unintentionally introduced when processing the image with a photo-editor tool or could be the result of a bad printing.

\section{Digital Beautification}

To obtain this alteration, we use LiftMagic [6], an instant cosmetic surgery and antiaging makeover tool that produces realistic image beautification. The tool presents a very simple web interface that allows to load an image and to simulate different plastic surgery treatments at different levels. It makes available 17 treatments: 16 local treatments (e.g., injectable for forehead, eyelid fold enhancement, lip augmentation, et al.) and one treatment integrating all the local ones. For each treatment, a specific selection bar allows to personalize the strength of the modification. In this alteration, we consider only the integrated treatment and three different strengths obtained by positioning the selection bar at three equidistant positions. We name the three levels 'low', 'medium' and ' high'. In other words, $p \in\{$ low, medium, high $\}$. Fig. 1 (e) presents the altered image after this alteration with $p=$ high.

\section{Effects of Alteration on Face Recognition Performance}

In this section we evaluate the effects of the above described alterations on face recognition accuracy. The experiments have been conducted with three different state-ofthe-art face recognition approaches: two commercial software (Neurotechnology VeriLookSDK 2.1 [7] (VL) and Luxand FaceSDK 4.0 [8] (LU)) and a SIFT-based matching algorithm [9] [10] (SI). The performance measured for the three systems on the unaltered database described below are good (see Fig. 2), so they constitute a good test bed to evaluate the effects of alterations: in particular the measured EER is $0.003 \%(\mathrm{VL}), 1.693 \%(\mathrm{LU})$ and $2.217 \%(\mathrm{SI})$.

\subsection{Database}

The choice of a proper face database is here an important issue. In fact, in the context of electronic documents, face images are expected to be high quality; hence, variations caused by illumination, expressions, poses, etc. should not be presented in the selected database. The selected database is AR face database [11]. This database con- 
sists of 4,000 frontal images taken under different conditions in two sessions, separated by two weeks. The images relevant to our study are well controlled and high quality images (with neutral expressions and good illumination), so the poses 1 and 14 are selected for the tests. We denote them as No1 and No14 respectively (see Fig. 3 for an example).

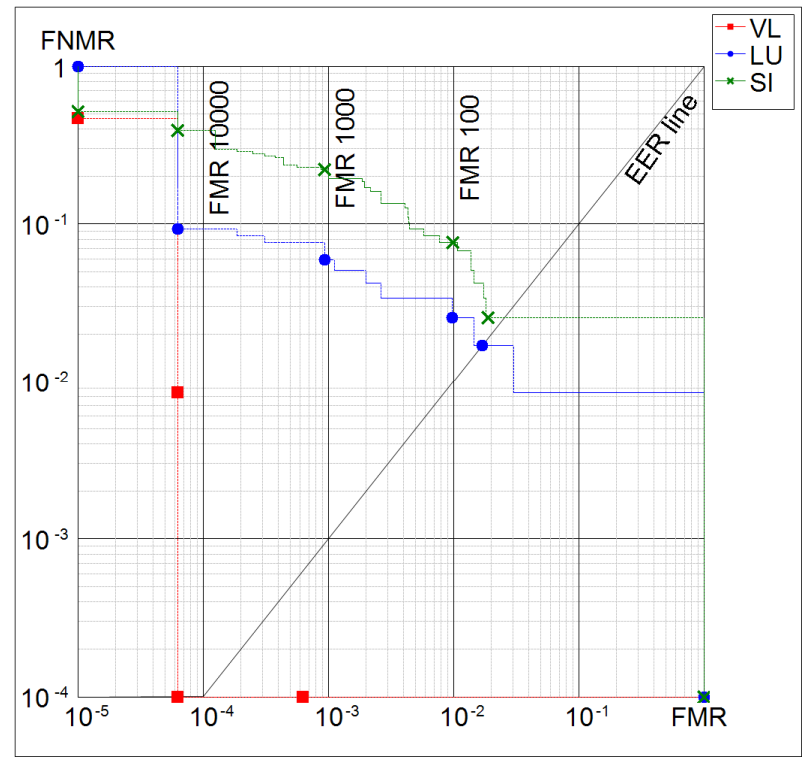

Fig. 2. DET curves of the three reference systems on the unaltered database
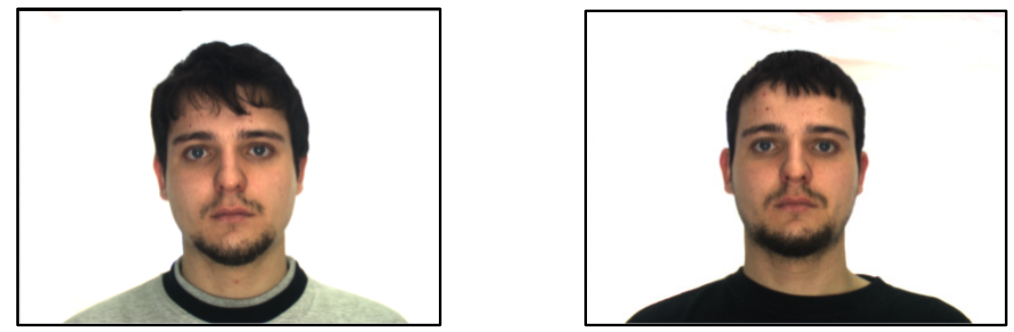

Fig. 3. Two unaltered images of the same subject in the AR database (pose 1 on the left, pose 14 on the right)

In our test we assume that the images No14 are used during enrollment (i.e., are stored in the e-documents), while the images No1 are used as probe (i.e., at the point of verification). The alterations are thus applied to images No14 to simulate the inclusion in the document of an altered image. 


\subsection{Face Recognition Results}

To evaluate the effects of the various alterations on face recognition accuracy, a systematic experimentation has been carried out. Starting from the original database, for each alteration, face images with different alteration strength have been generated by modifying the original images with different transformations (see Section 2).

The performance evaluation of face recognition algorithms is based on a set of genuine and impostor recognition attempts. In a genuine recognition attempt, two face images of the same individual are compared, while in an impostor attempt, two images from different persons are compared. In each genuine/impostor attempt, the first image is supposed to have been acquired during an 'enrollment' stage (and included into the document) and the second one during a 'verification' stage. The following performance indicators are used: False Non-Match Rate (FNMR) at a False Matching Rate $(\mathrm{FMR})$ of $1 \%\left(\mathrm{FMR}_{100}\right)$ and $1 \%$ o $\left(\mathrm{FMR}_{1000}\right)[12]$.

In the following definitions, each database $D B$ consists of two sets of face images: $D B_{e}$ (acquired during enrollment) and $D B_{v}$ (acquired during verification).

The original database (without alterations) is denotes as $D B^{O}=\left\{D B_{e}^{O}, D B_{v}^{O}\right\}$. $D B_{e}^{O}$ is made of all the original No14s of 120 subjects, while $D B_{v}^{O}$ is composed of all the original No1s (of 134 subjects). For genuine attempts, each No14 is compared against the No1 of the same subject; since only 118 subjects have both pose 1 and 14 , the number of genuine attempts is 118 . For impostor attempts, the No14 of one subject and all the No1 of the other subjects are compared. Hence, the total number of impostor attempts is 15962.

As to the altered databases, for a given alteration $a$ let $D B_{a}^{p}=\left\{\left(D B_{e}\right)_{a}^{p}, D B_{v}^{O}\right\}$ be a database that simulates enrollment face images reporting alteration $a$ with a strength of $p$. For genuine attempts, the original No1 and the altered No14 from the same subject are compared. Impostor attempts are the same as in the original database $D B^{O}$.

The results of the barrel distortion are reported in Fig. 4. It can be observed that both $\mathrm{FMR}_{100}$ and $\mathrm{FMR}_{1000}$ change slightly and irregularly as the degree of barrel distortion increases for LU and SI, while there is no significant performance change for VL. Overall this alteration has no noticeable effects on the recognition accuracy.

Fig. 5 and Fig. 6 illustrate the results of the vertical contraction and extension respectively. For both $\mathrm{FMR}_{100}$ and $\mathrm{FMR}_{1000}$, as the strength of the alterations increases, the accuracy of LU significantly decreases. SI shows a less noticeable performance drop than LU, while there is no significant performance change for VL.

Finally the results of the digital beautification are reported in Fig. 7. For both $\mathrm{FMR}_{100}$ and $\mathrm{FMR}_{1000}$, this alteration produces a performance drop for all the three system (even if LU shows a less noticeable reduction of the recognition accuracy).

Overall the experimental results show that the barrel alteration does not affects significantly the recognition accuracy. This is probably due to the fact that in the central part of the image containing the face, the barrel distortion produces simply a sort of scaling effect, which is well handled by the algorithms analyzed.

Aspect ratio alteration is critical for some approaches (for instance the vertical contraction at the maximum strength causes a performance drop of $\mathrm{FMR}_{1000}$ of about 11 times for LU) while it is just slightly disturbing other systems. In particular, we believe that face recognition based on local features only are quite insensitive to global geometric changes. 
Finally, alteration such as digital beautification, when applied with high strength, produce marked performance drop to all the system tested.
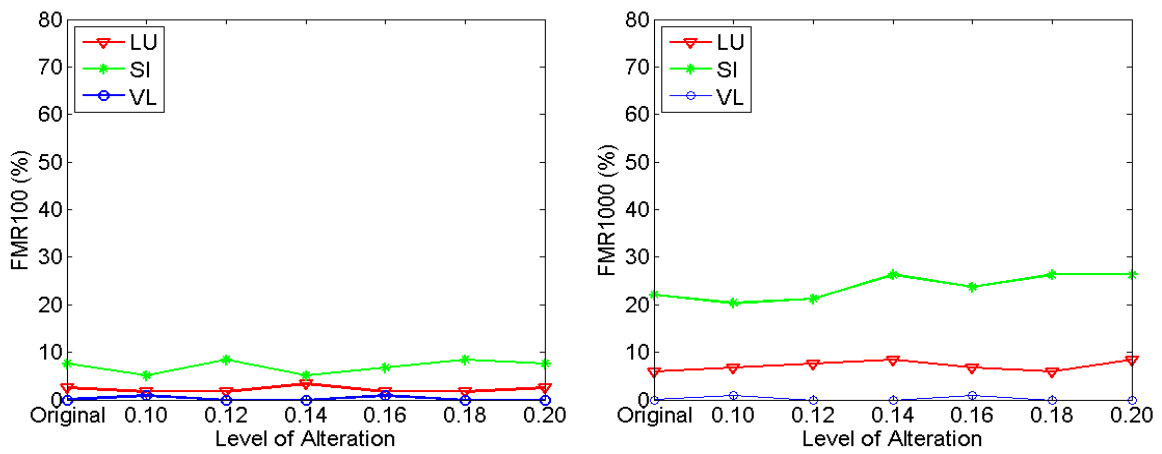

Fig. 4. Performance comparison before and after barrel distortion: $\mathrm{FMR}_{100}$ (left) and $\mathrm{FMR}_{1000}$ (right)
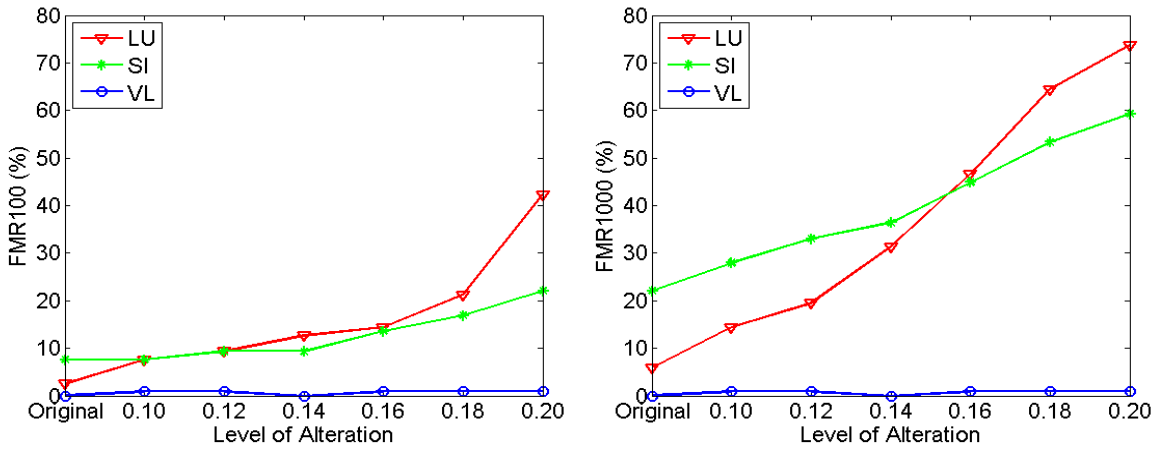

Fig. 5. Performance comparison before and after vertical contraction: $\mathrm{FMR}_{100}$ (left) and $\mathrm{FMR}_{1000}$ (right)
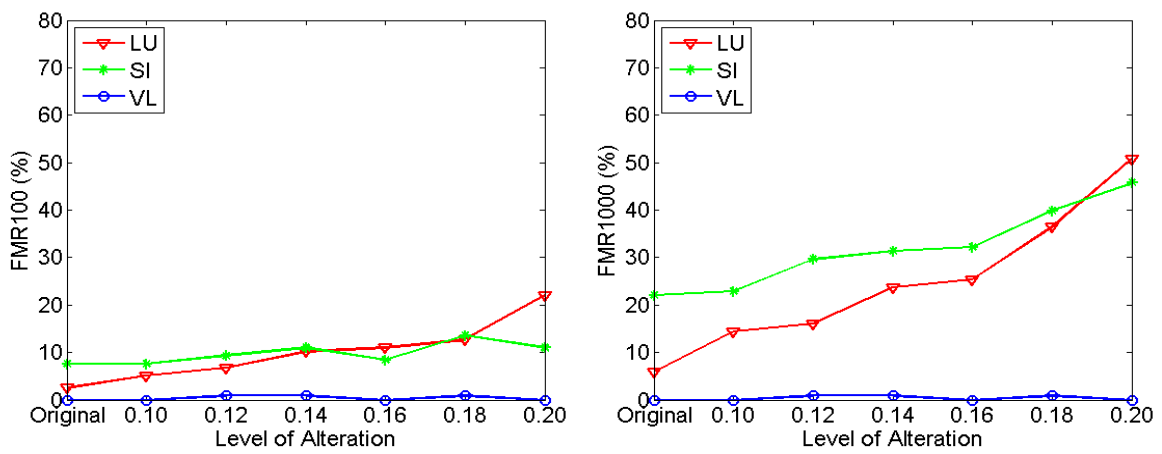

Fig. 6. Performance comparison before and after vertical extension: $F M R_{100}$ (left) and FMR ${ }_{1000}$ (right) 

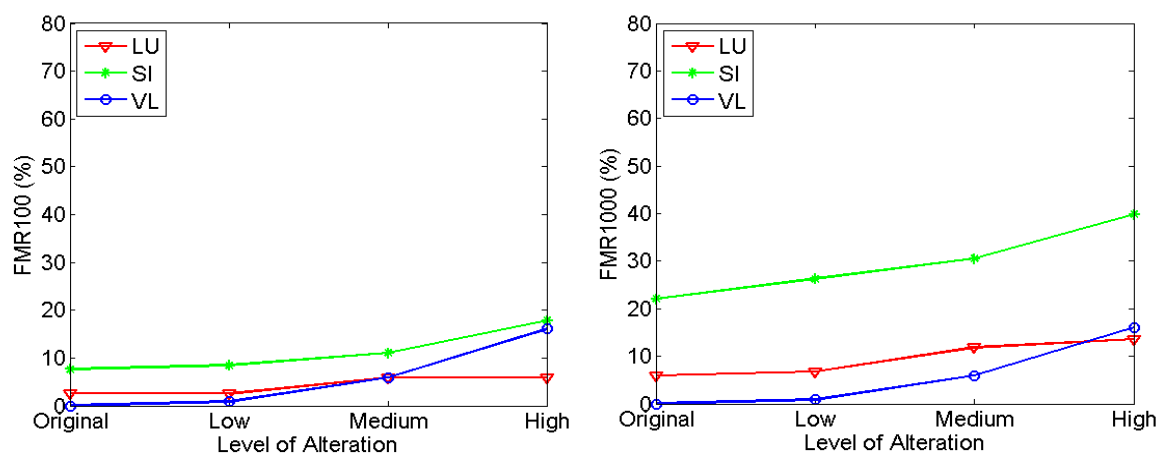

Fig. 7. Performance comparison before and after digital beautification: $F_{100}$ (left) and $\mathrm{FMR}_{1000}$ (right)

\section{Conclusions}

The outcome of this study is that state-of-the-art algorithms are able to overcome limited digital alterations but are sensitive to more relevant modifications, thus suggesting that proper countermeasures have to be taken to avoid storing digitally altered photo in eMRTD.

In particular we suggest that authorities issuing e-documents, based on printed ID photos provided by citizens, carefully check these photos to detect intentional or unintentional alterations. To this purpose the officer workstation could be equipped with a software supporting the operator in comparing the scanned version of the ID photo with the live acquired face image. Automatic face recognition system (possibly based on both global and local features) could be used to issue warning in case of low matching scores and a graphical superimposition of the two face image could easily reveal to the officer the presence of alteration.

Acknowledgment. The work leading to these results has received funding from the European Community's Framework Programme (FP7/2007-2013) under grant agreement $\mathrm{n}^{\circ} 284862$.

\section{References}

[1] Bourlai, T., Ross, A., Jain, A.K.: On matching digital face images against passport photos. In: 2009 International Conference on Biometrics, Identity and Security (BIdS), pp. 1-10 (2009)

[2] Bourlai, T., Ross, A., Jain, A.K.: Restoring degraded face images for matching faxed or scanned photos. IEEE Transactions on Information Forensics and Security 6(2), 371384 (2011)

[3] Biometric Deployment of Machine Readable Travel Documents. ICAO (2004)

[4] ISO/IEC 19794-5, Information technology - Biometric data interchange formats - Part 5: Face image data (2011) 
[5] Vass, G., Perlaki, T.: Applying and removing lens distortion in post production. In: Proceedings of 2nd Hungarian Conference on Computer Graphics and Geometry (2003)

[6] LiftMagic - Instant cosmetic surgery and anti-aging makeover tool (2013), http: //makeovr.com/liftmagic

[7] Neurotechnology Inc. Neurotechnology web site (2013), http: / / www . neurotechnology.com/

[8] Luxand Inc. Luxand Web Site (2013), http://luxand.com

[9] Lowe, D.G.: Distinctive Image Features from Scale-Invariant Keypoints. International Journal of Computer Vision 60(2), 91-110 (2004)

[10] Bicego, M., Grosso, A., Tistarelli, M.: On the Use of SIFT Features for Face Authentication. In: Conference on Computer Vision and Pattern Recognition Workshop, CVPRW 2006, p. 35 (2006)

[11] Martinez, A.M., Benavente, R.: The AR face database. Computer Vision Center, CVC Technical Report (1998)

[12] Maltoni, D., Maio, D., Jain, A.K., Prabhakar, S.: Handbook of Fingerprint Recognition, 2nd edn. Springer, New York (2009) 